\title{
DESIGN COMO INOVAÇÃO EM SUSTENTABILIDADE: uma revisão sistemática da literatura.
}

\author{
ALANO, Agda Bernardete. Mestranda \\ Pós-Graduação em Design e Expressão Gráfica-UFSC. \\ agda.alano@gmail.com \\ FIGUEIREDO, Luiz Fernando Gonçalves de. Dr. \\ Pós-Graduação em Design e Expressão Gráfica -UFSC. \\ lffigueiredo2009@gmail.com
}

\begin{abstract}
Resumo: O desenvolvimento de produto enfrenta desafios de integração das questões ambientais e, muitas vezes, as ações são tomadas de forma reativa, resultando em uma abordagem retrospectiva e insuficiente. Para pequenas e médias empresas o desafio ainda é maior, considerando a falta de experiência e o custo de informação.

Há um corpo estabelecido de pesquisa para promover a sustentabilidade ambiental em produtos e processos, que neste estudo é tratado a partir de uma revisão sistemática de literatura, cuja apresentação se dá nas relações entre a inovação e o desenvolvimento de produtos com foco na sustentabilidade. Buscou-se, nessa pesquisa, estudos que tratassem do design a partir de ações estratégicas, assim, foi considerada uma busca por estudos que apresentassem ferramentas e métodos disponíveis para promover eco inovação em casos de pequenos e médios empreendimentos (PMEs).

Palavras-chave: Inovação, Sustentabilidade, Design.
\end{abstract}

Abstract:Product development faces challenges of integrating environmental issues, and often actions are taken in a reactive way, resulting in a retrospective approach and insufficient. For small and medium businesses the challenge is even greater considering the lack of experience and information costs.

There is an established body of research to promote environmental sustainability in products and processes, which in this study is treated from a systematic review of the literature, and presents links between innovation and product development with a focus on sustainability. We sought in this research studies that addressed the design from strategic actions, so we took into account in studies that showed search tools and methods available to promote eco-innovation in SMEs cases

Keywords: Innovation, Sustainability, Design. 


\section{INTRODUÇÃO}

O design compreende a inovação em uma organização como possibilidade que, neste estudo, é tratada a partir de sua relação com a sustentabilidade.

Assim, são considerados como fatores de análise, não somente a perspectiva do produto, mas o design enquanto processo, os quais são assim analisados desde a infraestrutura técnica, até os atores que fazem parte da cadeia de produtos ou serviços.

Levando em conta a complexidade de uma abordagem que trata da sustentabilidade em design, destaca-se o eixo ambiental, com base em propostas atuais de inovação, por meio de ferramentas capazes de contribuir com uma produção limpa que possa ser aplicada em casos de pequenos empreendimentos, visando, nesse contexto, a ampliação de valores, não somente por meio de resultados econômicos, mas também de aspectos sociais.

Assim, a sustentabilidade, vista como valor, é tratada em uma visão sustentável que, como fenômeno social, gera uma mudança qualitativa em produtos e processos, sendo obtida através da criação de novos conhecimentos e percebida como novo valor por uma rede social (BALDWIN et al., 2005). Além disso, segundo Mozota (2003), a inovação justifica o desempenho na perspectiva da gestão do design, a qual anteriormente era vista como um ator externo para a diferenciação da empresa, tornando-se, dessa forma, um ator interno no processo organizacional (MOZOTA, 2003). Manzini (2008, p. 14) salienta a importância de "[...] desenvolver a capacidade de reconhecer o valor de um caso de inovação social sustentável quanto a fomentar a habilidade dos designers em projetar um conjunto de soluções capaz de aperfeiçoá-lo e de reproduzi-lo em diversos contextos".

\section{DESENVOLVIMENTO}

Este estudo realizou uma revisão sistemática da literatura, a fim de conhecer o constructo Inovação em sua relação com a sustentabilidade; e como o design participa deste contexto para geração de valor.

Nesta busca, considera-se como unidade de análise a aplicação da inovação em sustentabilidade em pequenos empreendimentos, os quais serão tratados nesta revisão a partir das PMEs.

Para a delimitação da pesquisa, lançou-se mão de uma busca na base de dados Scopus, a qual foi escolhida por ser uma base multidisciplinar que possibilita levantamentos por palavras-chave, resumos e títulos das publicações. Em Freire (2010, p.45) encontra-se que "a base Scopus é hoje a maior base de resumos e referências bibliográficas de literatura científica, revisada por pares, permitindo uma visão multidisciplinar e integrada de fontes relevantes para a pesquisa bibliográfica sistemática".

A busca eletrônica nas bases de dados Scopus limitou-se, em um primeiro momento, à busca pelas palavras Innovation e Sustainability, restringindo-se à data de publicações a partir de 2007. Nesse contexto, foram encontrados 2987 artigos. Diante do interesse de um refinamento e aproximação com o foco de estudo, incluiu-se o termo Design, o que resultou em 783 artigos.

Durante a busca, observou-se a reincidência do constructo Eco nos estudos que tratavam do design como inovação em produtos e processos, e por isso, foi substituída 
a palavra "Sustainability" pelo constructo Eco, com data de publicação também considerada a partir de 2007.

Essa alteração resultou em 221 artigos, sendo que parte desses estudos tratava do design como abordagem metodológica (desenho de pesquisa). Para esse caso, utilizou-se como refinamento no quadro de busca a exclusão (AND NOT) dos termos "design/methodology/approach", e foram excluídos dezenove estudos, resultando em 202 artigos selecionados que resultaram numa amostra não probabilística por conveniência.

A imagem abaixo representa como os critérios foram determinados utilizandose a interface de busca da base de dados Scopus.

Figura 1: Quadro das buscas

\section{Scopus}

\section{Search | Alerts | My list | Settings}

Scopus to cease support of Internet Explorer 7 (IET)

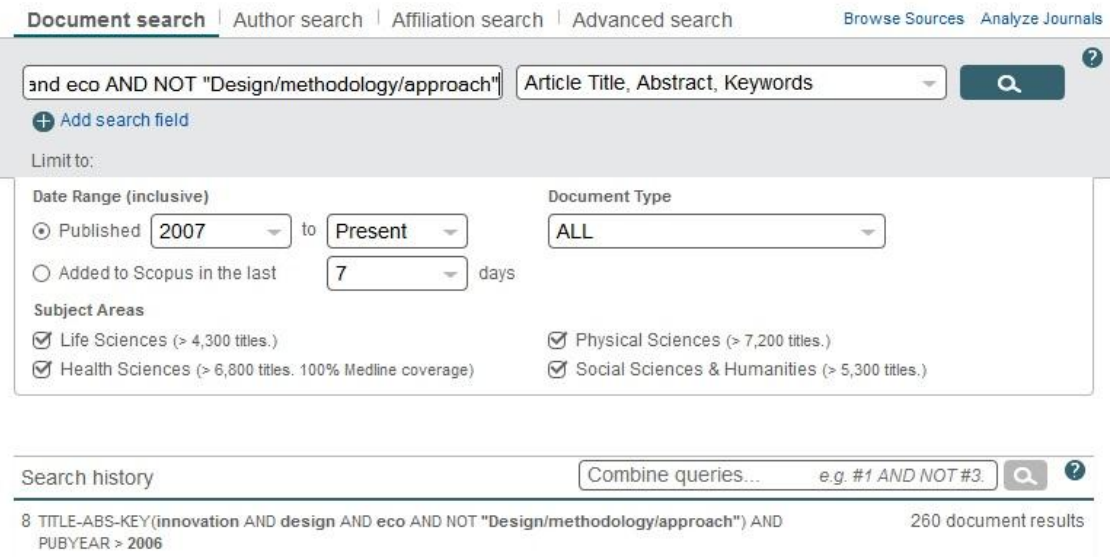

Fonte: Base de dados Scopus, 2014

O seguinte quadro apresenta o resultado de busca de acordo com a inserção dos critérios.

Quadro 1: Quadro de seleção dos artigos

\begin{tabular}{|c|c|c|}
\hline Critérios de busca & Observação & $\begin{array}{c}\text { № de } \\
\text { Artigos }\end{array}$ \\
\hline Palavras-chave de busca & "Innovation" and "Sustainability" & 2987 \\
\hline Palavras-chave de busca & "Innovation" and "Sustainability" and & 783 \\
\hline "Design" & "Innovation" and "Design" and "Eco" & 221 \\
\hline Exclusão de design como metodologia & "Design/methodology/approach" 2008 & 202 \\
\hline
\end{tabular}

Fonte: Elaborado pelos autores, 2014 


\subsection{Revisão sistemática: análises iniciais}

A partir dos 202 artigos selecionados utilizando como critério das palavras "Innovation and Design and Eco", na segunda busca, destaca-se, de acordo com o quadro abaixo, um crescente número nas publicações a partir de 2010.

Figura 2: Análises iniciais

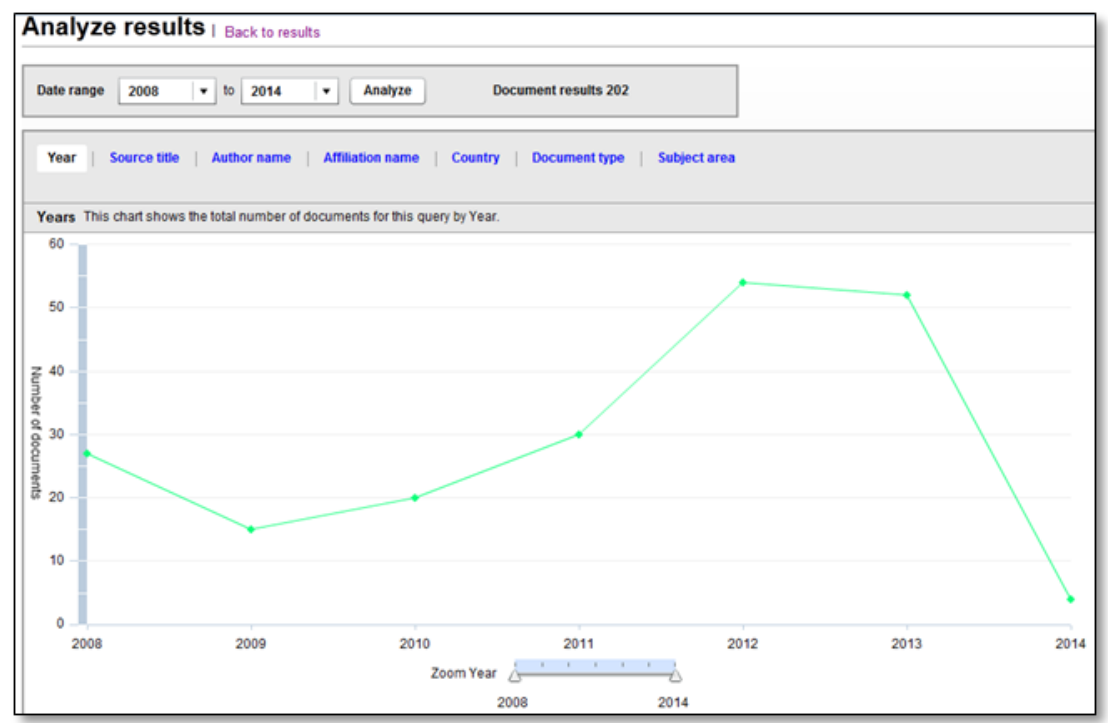

Fonte: Base de dados Scopus, 2014

Quadro 2: Artigos mais relevantes

\begin{tabular}{|c|c|c|c|}
\hline Autores & Titulo do artigo & Data & $\begin{array}{c}\text { no de } \\
\text { citações }\end{array}$ \\
\hline Ren, H.; Gao, W., & $\begin{array}{l}\text { "Um modelo MILP para o plano e avaliação de } \\
\text { sistemas de energia distribuída integrada" }\end{array}$ & 2010 & 63 \\
\hline $\begin{array}{l}\text { Zabalza Bribián, I.; } \\
\text { Valero Capilla, } \\
\text { A.;Aranda Usón, A. }\end{array}$ & $\begin{array}{c}\text { "A avaliação do ciclo de vida dos materiais de } \\
\text { construção: Análise comparativa de energia e os } \\
\text { impactos ambientais e avaliação do potencial de } \\
\text { melhoria da eco-eficiência" }\end{array}$ & 2011 & 50 \\
\hline $\begin{array}{l}\text { Carrillo-Hermosilla, J.; } \\
\text { Del Río, P., Könnölä, }\end{array}$ & $\begin{array}{l}\text { "A diversidade de eco-inovações: Reflexões a partir } \\
\text { de estudos de caso selecionados" }\end{array}$ & 2010 & 28 \\
\hline $\begin{array}{l}\text { Zhang, T.; Gensler, S.; } \\
\text { Garcia, R., }\end{array}$ & $\begin{array}{l}\text { "Um estudo da difusão de veículos movidos a } \\
\text { combustíveis alternativos: Uma abordagem de } \\
\text { modelagem baseada em agentes" }\end{array}$ & 2011 & 21 \\
\hline $\begin{array}{l}\text { Petraru, M.; } \\
\text { Gavrilescu, M. }\end{array}$ & $\begin{array}{l}\text { "A prevenção da poluição, a chave para a } \\
\text { sustentabilidade econômica e ambiental" }\end{array}$ & 2010 & 19 \\
\hline Smith, M.; Crotty, J. & $\begin{array}{l}\text { "A regulamentação ambiental e a inovação design } \\
\text { ecológico na indústria automotiva no Reino Unido" }\end{array}$ & 2008 & 17 \\
\hline Yang, C.J.; Chen, J.L. & "Acelerar projeto de eco inovação preliminar de & 2011 & 15 \\
\hline
\end{tabular}




\begin{tabular}{|c|c|c|c|}
\hline & $\begin{array}{c}\text { produtos que integra raciocínio baseado em casos e } \\
\text { método TRIZ" }\end{array}$ & & \\
\hline Niinimäki, K.; Hassi, L. & $\begin{array}{l}\text { "Emergentes estratégias de design na produção } \\
\text { sustentável e consumo de têxteis e vestuário" }\end{array}$ & 2011 & 14 \\
\hline $\begin{array}{l}\text { Cellura, M.; Longo, S., } \\
\text { Mistretta, M. }\end{array}$ & $\begin{array}{l}\text { "A aplicação da análise de decomposição estrutural } \\
\text { para avaliar o consumo energético e das emissões } \\
\text { atmosféricas mudanças indiretos relacionados à } \\
\text { italiana, consumo das famílias" }\end{array}$ & 2012 & 11 \\
\hline $\begin{array}{l}\text { Santolaria, M.; Oliver- } \\
\text { Sol, J.; Gasol, C.M.; } \\
\text { Morales-Pinzón, T.; } \\
\quad \text { Rieradevall, J. }\end{array}$ & $\begin{array}{l}\text { Eco-design na inovação orientada empresas: } \\
\text { percepção, previsões e os principais } \\
\text { impulsionadores da integração. o exemplo espanhol }\end{array}$ & 2011 & 10 \\
\hline $\begin{array}{l}\text { Elmquist, M., } \\
\text { Segrestin, B. }\end{array}$ & $\begin{array}{l}\text { "O desenvolvimento sustentável através de } \\
\text { projetos inovadores: as lições da KCP método } \\
\text { experimentado com uma empresa automotiva" }\end{array}$ & 2009 & 10 \\
\hline $\begin{array}{l}\text { Bocken, N.M.P.; } \\
\text { Allwood, J.M.; Willey, } \\
\text { A.R., King, J.M.H. }\end{array}$ & $\begin{array}{l}\text { "Desenvolvimento de uma ferramenta de eco- } \\
\text { ideação para identificar opções de redução de } \\
\text { emissões de gases de efeito estufa por etapas, para } \\
\text { bens de consumo". }\end{array}$ & 2011 & 9 \\
\hline Yang, C.J.; Chen, J.L. & $\begin{array}{l}\text { "Prevendo o projeto de eco-produtos, integrando } \\
\text { padrões de evolução TRIZ com CBR e métodos de } \\
\text { ACV simples" }\end{array}$ & 2012 & 7 \\
\hline $\begin{array}{l}\text { Negny, S.; Belaud, J.P.; } \\
\text { Cortes Robles, G.; } \\
\text { Roldan Reyes, E.; } \\
\text { Ferrer, J.B. }\end{array}$ & $\begin{array}{l}\text { "Rumo a um método de eco inovação baseado em } \\
\text { uma melhor utilização dos recursos: aplicação a } \\
\text { processo químico projeto preliminar" }\end{array}$ & 2011 & 6 \\
\hline $\begin{array}{l}\text { Yeh, C.H.; Huang, } \\
\text { J.C.Y.; Yu, C.K.; }\end{array}$ & $\begin{array}{l}\text { "A integração de quatro fases QFD e TRIZ no } \\
\text { produto R \& D: Um estudo de caso notebook " }\end{array}$ & 2012 & 6 \\
\hline $\begin{array}{l}\text { Boks, C.; McAloone, } \\
\text { T.C. }\end{array}$ & $\begin{array}{c}\text { "Transições em pesquisa de design de produto } \\
\text { sustentável" }\end{array}$ & 2009 & 6 \\
\hline $\begin{array}{l}\text { Deutz, P.; McGuire, M.; } \\
\text { Neighbour, G. }\end{array}$ & $\begin{array}{l}\text { "A prática de Eco-design no contexto de um } \\
\text { processo de design estruturado: um estudo } \\
\text { empírico interdisciplinar de fabricantes do Reino } \\
\text { Unido" }\end{array}$ & 2013 & 4 \\
\hline $\begin{array}{l}\text { Giustozzi, F.; Toraldo, } \\
\text { E.; Crispino, M. }\end{array}$ & $\begin{array}{l}\text { "Pavimentos reciclados aeroporto para se alcançar } \\
\text { a sustentabilidade ambiental: um estudo de caso } \\
\text { italiano" } 2011\end{array}$ & 2012 & 4 \\
\hline
\end{tabular}

Fonte: Base de dados Scopus, 2014

Muitos estudos, apesar de tratarem o design como uma das variáveis, focalizam os resultados na sustentabilidade com foco nos recursos energéticos; também um número representativo de estudos apresentou a engenharia como área de estudo. Apesar de esses enfoques representarem de forma significativa elementos de inovação em sustentabilidade, este estudo limita-se a encontrar temas relacionados à inovação como ação estratégica e, ainda, discutir como o design é aplicado nesses casos. Diante disso, foram excluídos os estudos com enfoque na engenharia e energia e delimitou-se 
um novo filtro na busca, para isso foram selecionados apenas artigos relacionados às seguintes palavras-chave: "Innovation", "Product design", "Sustainability", "Design" e "Eco-design". Então, foram randomizados 37 artigos.

\section{FERRAMENTAS DE AVALIAÇÃO}

Dentre essa amostra de 37 artigos, utilizaram-se como critério de seleção, artigos que contivessem em suas palavras-chave termos associados à: Sistemas colaborativos, Teoria Triz, LCA, SMEs, Aprendizagem organizacional, plataformas de suporte, redes de valor, o que resultou em uma amostra de 22 artigos randomizados.

Quadro 3: Quadro dos artigos selecionados

\begin{tabular}{|c|c|}
\hline KEYWORDS & TÍTULO DO ARTIGO \\
\hline $\begin{array}{l}\text { Eco-design; ICT; LCA; Product (re)design; } \\
\text { Web services }\end{array}$ & $\begin{array}{l}\text { Integrating services and tools in an ICT platform to support } \\
\text { eco-innovation in SMEs }\end{array}$ \\
\hline $\begin{array}{l}\text { Biomass; CSP; Eco-innovation design; Multi } \\
\text { contradiction; TRIZ }\end{array}$ & Eco-innovative design method for process engineering \\
\hline TRIZ; innovation & $\begin{array}{l}\text { Proposition of an eco-design approach for an easy } \\
\text { appropriation by companies }\end{array}$ \\
\hline $\begin{array}{l}\text { Competencies; Eco-innovation; Innovation } \\
\text { space; Life cycle thinking; Value chain; } \\
\text { Value star }\end{array}$ & 7.Eco-innovation in the value chain \\
\hline $\begin{array}{l}\text { Eco-efficient; Green Design; Light Emitting } \\
\text { Diode (LED); Product Innovation; Theory of } \\
\text { Inventive Problem Solving (TRIZ) }\end{array}$ & $\begin{array}{l}\text { The development of an innovative design process for eco- } \\
\text { efficient green products }\end{array}$ \\
\hline $\begin{array}{l}\text { : Eco-innovation; Extended producer } \\
\text { responsibility; Policy instruments; } \\
\text { Simulation models }\end{array}$ & $\begin{array}{l}\text { Extended producer responsibility instruments and } \\
\text { innovation in eco-design: An exploration through a } \\
\text { simulation model }\end{array}$ \\
\hline $\begin{array}{l}\text { actions for eco-innovation; distribution } \\
\text { phase; eco-innovation; Eco-MAL'IN; } \\
\text { functional and energy analysis; sustainable } \\
\text { development; Triz theory }\end{array}$ & $\begin{array}{l}\text { Eco-innovative method to improve the distribution phase of } \\
\text { product }\end{array}$ \\
\hline $\begin{array}{l}\text { Design for environment; Eco-design; New } \\
\text { product development process; Product } \\
\text { attachment; Product innovation; Product } \\
\text { value; Sustainability; Sustainable design }\end{array}$ & $\begin{array}{l}\text { Key aspects of product attraction: A focus on eco- } \\
\text { friendliness }\end{array}$ \\
\hline $\begin{array}{l}\text { Design and innovations; Design Principles; } \\
\text { Eco effectiveness; Eco-efficiency; Natural } \\
\text { systems }\end{array}$ & Design principles for green ergonomics \\
\hline
\end{tabular}




\begin{tabular}{|c|c|}
\hline $\begin{array}{l}\text { Collaborative work; Design process; Eco- } \\
\text { innovation; Ecodesign integration; } \\
\text { Ecodesign tools; Environmental } \\
\text { performance; France; Green products; } \\
\text { Industrial ecology; Learning; Product } \\
\text { design; Small and medium-sized } \\
\text { enterprises; SMEs; Strategy; Sustainable } \\
\text { product development; Trojan horse }\end{array}$ & $\begin{array}{l}\text { An exploratory study for the long-term integration of } \\
\text { ecodesign in SMEs: The environmental Trojan horse } \\
\text { strategy }\end{array}$ \\
\hline $\begin{array}{l}\text { Early design stages; Eco-design; } \\
\text { Estimations; Industrial ecology; Life cycle } \\
\text { assessment (LCA); Product development }\end{array}$ & $\begin{array}{l}\text { Estimating Environmental Behavior Without Performing a } \\
\text { Life Cycle Assessment }\end{array}$ \\
\hline $\begin{array}{l}\text { Knowledge management; Lifestyle design; } \\
\text { Product-service; Reverse logistics; Service } \\
\text { engineering }\end{array}$ & Knowledge management and eco-design scopes \\
\hline $\begin{array}{l}\text { Automotive; Eco-efficiency; EU End of Life } \\
\text { Vehicles Directive; Innovation; UK }\end{array}$ & $\begin{array}{l}\text { Environmental regulation and innovation driving ecological } \\
\text { design in the UK automotive industry }\end{array}$ \\
\hline $\begin{array}{l}\text { Eco-design; Eco-innovation; PGM; } \\
\text { Precautionary principle; REE; Resource } \\
\text { depletion; Sustainability ethics; Sustainable } \\
\text { resource management }\end{array}$ & $\begin{array}{l}\text { Material Scarcity: A Reason for Responsibility in Technology } \\
\text { Development and Product Design }\end{array}$ \\
\hline $\begin{array}{l}\text { Eco design; environmental sustainability; } \\
\text { green innovation; green product innovation }\end{array}$ & $\begin{array}{l}\text { Linking green product innovation, technological and human } \\
\text { resource capabilities: A conceptual model }\end{array}$ \\
\hline $\begin{array}{l}\text { Bio-ispired design approach; Biomimetic } \\
\text { guidelines; Sustainable design }\end{array}$ & Biomimetic and sustainable design: A virtuous relationship \\
\hline $\begin{array}{l}\text { Complex industrials system; Creativity; Eco- } \\
\text { design; Eco-design strategy wheel; Eco- } \\
\text { innovation; R\&D project portfolio }\end{array}$ & $\begin{array}{l}\text { Identification and selection of eco-innovative R\&D projects } \\
\text { in complex systems industries }\end{array}$ \\
\hline $\begin{array}{l}\text { Alternative materials; Development; Eco- } \\
\text { efficiency; Industrial Ecology; Innovation }\end{array}$ & $\begin{array}{l}\text { Multicriteria decision making techniques in the } \\
\text { selection of structural components from wood } \\
\text { technology, for construction of social housing in } \\
\text { Venezuela }\end{array}$ \\
\hline $\begin{array}{l}\text { Design; environmental tourism; } \\
\text { experiences; organic farms; transition }\end{array}$ & $\begin{array}{l}\text { Experiencing organic farms and food by regional tourism } \\
\text { innovation }\end{array}$ \\
\hline $\begin{array}{l}\text { Data quality; Industrial ecology; } \\
\text { Information and communications } \\
\text { technology (ICT); Life cycle assessment } \\
\text { (LCA); Ontology; Semantic web }\end{array}$ & $\begin{array}{l}\text { Application of Product Data Technology Standards to LCA } \\
\text { Data }\end{array}$ \\
\hline
\end{tabular}




\begin{tabular}{|l|l|}
\hline $\begin{array}{l}\text { Circular economy; Ecological footprint; } \\
\text { Innovation; Resource productivity; } \\
\begin{array}{l}\text { Services; Sustainable infrastructure; } \\
\text { Systems }\end{array}\end{array}$ & $\begin{array}{l}\text { 36.Sustainable urban infrastructure in China: Towards a } \\
\text { Factor } 10 \text { improvement in resource productivity through } \\
\text { integrated infrastructure systems }\end{array}$ \\
\hline $\begin{array}{l}\text { Agroforestry; Family farmers; Participatory } \\
\text { experimentation; Systematization }\end{array}$ & $\begin{array}{l}\text { Learning by doing: A participatory methodology for } \\
\text { systematization of experiments with agroforestry systems, } \\
\text { with an example of its application }\end{array}$ \\
\hline
\end{tabular}

Fonte: Base de dados Scopus, 2014

A fim de verificar como o design e a inovação se relacionavam com a sustentabilidade, utilizou-se a análise por meio de mapas conceituais com o intuito de destacar nos estudos os conceitos, relações, sistemas e proposições.

A figura abaixo representa uma análise de um dos estudos avaliados, sendo consideradas as relações de conceitos, nas quais é possível destacar elementos chaves em que, em uma análise comparativa com o mapa de outros estudos, pode evidenciar como o design participa, enquanto gestão e processo, e a sua relação com o ecossistema e a inovação.

Figura 3: Mapa Conceitual

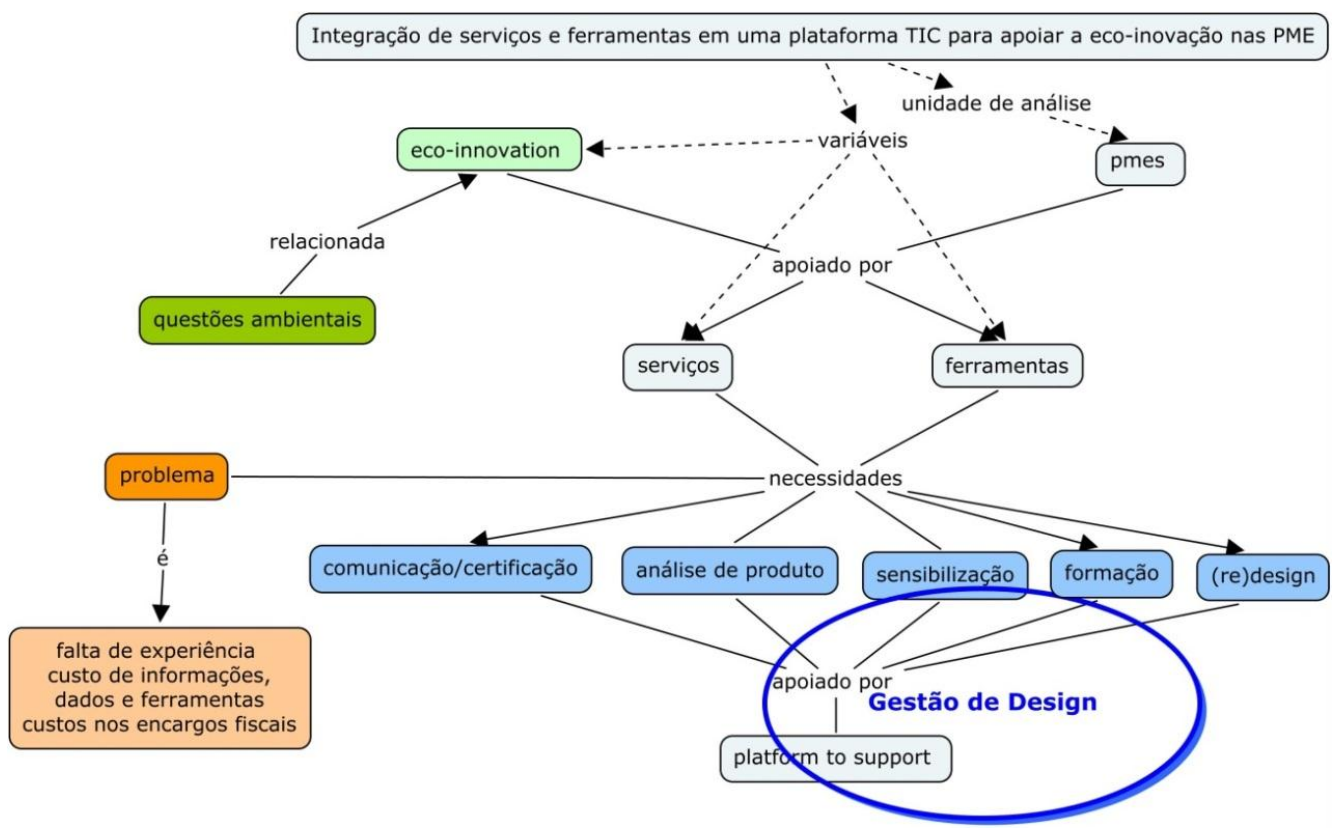

Fonte: Elaborado pelos autores, 2014

De acordo com o mapa, no estudo aplicado a uma PME, é possível identificar como as questões ambientais se relacionam ao estudo definido pelo constructo ecoinnovation, sendo apoiado por uma plataforma de suporte. O design aqui é representado por ações estratégicas na análise de produto, sensibilização, formação, (re) design e comunicação/certificação, a fim de solucionar problemas relativos à falta de experiência ao custo de informações, à necessidade de dados e de ferramentas e encargos fiscais. Como resultado apresenta como instrumento uma plataforma de 
suporte. Apesar do estudo não relacionar a gestão organizacional à gestão de design, especificamente, é possível destacar tal relação, a partir da identificação de ferramentas que auxiliam as ações no processo de planejamento do produto-serviço.

De acordo com a análise desse estudo, foram aplicados aos demais 21 estudos a verificação dos conceitos: questões ambientais, design e inovação para identificar aproximações com a gestão de design e a sustentabilidade, o que resultou em dez artigos selecionados.

Quadro 4: artigos escolhidos

\begin{tabular}{|c|c|c|}
\hline Título do artigo & KEYWORDS: & Autores \\
\hline $\begin{array}{l}\text { Integrating services and tools in an ICT } \\
\text { platform to support eco-innovation in SMEs }\end{array}$ & $\begin{array}{l}\text { Eco-design; ICT; LCA; } \\
\text { Product (re)design; Web } \\
\text { services }\end{array}$ & $\begin{array}{l}\text { Buttol, P., Buonamici, } \\
\text { R., Naldesi, L., Rinaldi, } \\
\text { C., Zamagni, A., } \\
\text { Masoni, P. }\end{array}$ \\
\hline $\begin{array}{c}\text { Eco-innovative design method for process } \\
\text { engineering }\end{array}$ & $\begin{array}{c}\text { Biomass; CSP; Eco- } \\
\text { innovation design; Multi } \\
\text { contradiction; TRIZ }\end{array}$ & $\begin{array}{l}\text { Ferrer, J.B.a, Negny, } \\
\text { S.a, Robles, G.C.b, Le } \\
\text { Lann, J.M.a }\end{array}$ \\
\hline $\begin{array}{c}\text { Proposition of an eco-design approach for an } \\
\text { easy appropriation by companies }\end{array}$ & TRIZ; innovation & $\begin{array}{l}\text { Cherifi, A.a b, } \\
\text { Gardoni, M.a, Tairi, } \\
\text { A.b }\end{array}$ \\
\hline Eco-innovation in the value chain & $\begin{array}{l}\text { Competencies; Eco- } \\
\text { innovation; Innovation } \\
\text { space; Life cycle thinking; } \\
\text { Value chain; Value star }\end{array}$ & $\begin{array}{l}\text { McAloone, T.C., } \\
\text { Mougaard, K., } \\
\text { Restrepo, J., Knudsen, } \\
\text { S. }\end{array}$ \\
\hline $\begin{array}{c}\text { Extended producer responsibility instruments } \\
\text { and innovation in eco-design: An exploration } \\
\text { through a simulation model }\end{array}$ & $\begin{array}{l}\text { Eco-innovation; Extended } \\
\text { producer responsibility; } \\
\text { Policy instruments; } \\
\text { Simulation models }\end{array}$ & Brouillat, E., Oltra, V. \\
\hline $\begin{array}{l}\text { An exploratory study for the long-term } \\
\text { integration of eco design in SMEs: The } \\
\text { environmental Trojan horse strategy }\end{array}$ & $\begin{array}{l}\text { Collaborative work; Design } \\
\text { process; Eco-innovation; } \\
\text { Ecodesign integration; } \\
\text { Ecodesign tools; } \\
\text { Environmental performance; } \\
\text { France; Green products; } \\
\text { Industrial ecology; Learning; } \\
\text { Product }\end{array}$ & Reyes, T.a , Millet, D.b \\
\hline $\begin{array}{c}\text { Environmental regulation and innovation } \\
\text { driving ecological design in the UK automotive } \\
\text { industry }\end{array}$ & $\begin{array}{l}\text { Eco-efficiency; EU End of } \\
\text { Life Vehicles Directive; } \\
\text { Innovation; }\end{array}$ & Smith, M.a , Crotty, J.b \\
\hline $\begin{array}{c}\text { Biomimetic and sustainable design: A virtuous } \\
\text { relationship }\end{array}$ & $\begin{array}{l}\text { A teoria da complexidade - } \\
\text { Bio-ispired design approach; } \\
\text { Biomimetic guidelines; } \\
\text { Sustainable design }\end{array}$ & Stoppa, M. \\
\hline $\begin{array}{l}\text { Experiencing organic farms and food by } \\
\text { regional tourism innovation }\end{array}$ & $\begin{array}{l}\text { design; environmental } \\
\text { tourism; experiences; } \\
\text { organic farms; transition }\end{array}$ & $\begin{array}{l}\text { Holm, J.a , Pedersen, } \\
\text { L.M.B.a, Sørensen, S.b }\end{array}$ \\
\hline
\end{tabular}




\begin{tabular}{|c|c|l|}
\hline $\begin{array}{c}\text { Learning by doing: A participatory } \\
\text { methodology for systematization of } \\
\text { experiments with agroforestry systems, with }\end{array}$ & $\begin{array}{c}\text { Agroforestry; Family } \\
\text { farmers; Participatory } \\
\text { an example of its application }\end{array}$ & $\begin{array}{c}\text { De Souza, H.N., } \\
\text { Cardoso, I.M., de Sá }\end{array}$ \\
& Systematization; & Mendonça, E., \\
& & Carvalho, A.F., de \\
& & Oliveira, G.B., Gjorup, \\
D.F., Bonfim, V.R.,". \\
\hline
\end{tabular}

Fonte: Elaborado pelos autores, 2014

No quadro abaixo, apresentam-se os estudos que mais se aproximaram com o foco da pesquisa, os quais estão classificados pelo grau de relevância do autor, bem como pela relação da sustentabilidade com seus termos afins, sendo considerado como quadro de análise, assim como unidades e ferramentas e processos utilizados como proposta nos estudos.

Quadro 5: Unidades de análise e ferramentas

\begin{tabular}{|c|c|c|c|c|}
\hline & Sustentabilidade & Unidades de análise & Ferramenta/Processo & Autores \\
\hline 1 & Eco-innovation & $\begin{array}{c}\text { Integração serviços } \\
\text { PMEs }\end{array}$ & Plataforma TIC & $\begin{array}{l}\text { Buttol, P., } \\
\text { Buonamici, R., } \\
\text { Naldesi, L., } \\
\text { Rinaldi, C., } \\
\text { Zamagni, A., } \\
\text { Masoni, P. }\end{array}$ \\
\hline 2 & $\begin{array}{c}\text { Eco-innovation } \\
\text { Eco-friendly }\end{array}$ & Process engineering & $\begin{array}{c}\text { Plataforma de suporte } \\
\text { (CAD) } \\
\text { Teoria TRIZ }\end{array}$ & $\begin{array}{l}\text { Ferrer, J.B.a, } \\
\text { Negny, S.a, } \\
\text { Robles, G.C.b, Le } \\
\text { Lann, J.M.a }\end{array}$ \\
\hline 7 & Eco-innovation & Cadeia de valor & $\begin{array}{c}\text { Análise descritiva, } \\
\text { barreiras, oportunidades, } \\
\text { mecanismos }\end{array}$ & $\begin{array}{l}\text { McAloone, T.C., } \\
\text { Mougaard, K., } \\
\text { Restrepo, J., } \\
\text { Knudsen, S. }\end{array}$ \\
\hline 16 & $\begin{array}{c}\text { Eco-design } \\
\text { Eco-innovation }\end{array}$ & PME & $\begin{array}{c}\text { Eco design } \\
\text { Cavalo de Tróia } \\
\text { ambiental (ETHS) }\end{array}$ & $\begin{array}{l}\text { Reyes, T.a, } \\
\text { Millet, D.b }\end{array}$ \\
\hline 22 & $\begin{array}{l}\text { Eco-efficiency; } \\
\text { Eco design }\end{array}$ & $\begin{array}{c}\text { Regulamentação } \\
\text { ambiental }\end{array}$ & $\begin{array}{l}\text { Inovação incremental } \\
\text { Desmaterialização } \\
\text { Dobers e Wolff (1999) }\end{array}$ & $\begin{array}{l}\text { Smith, M.a, } \\
\text { Crotty, J.b }\end{array}$ \\
\hline 25 & $\begin{array}{l}\text { Eco-efficiency } \\
\text { Regeneração }\end{array}$ & Design sustentável & Teoria da complexidade) & Stoppa, M. \\
\hline 30 & Eco & $\begin{array}{l}\text { Turismo regional } \\
\text { Orgânica instrutivo }\end{array}$ & $\begin{array}{l}\text { Aprendizagem cultural } \\
\text { Design de experiência }\end{array}$ & $\begin{array}{l}\text { Holm, J.a, } \\
\text { Pedersen, } \\
\text { L.M.B.a, } \\
\text { Sørensen, S.b }\end{array}$ \\
\hline 37 & Eco-friendly & $\begin{array}{c}\text { Sistemas locais } \\
\text { Interações reflexivas }\end{array}$ & $\begin{array}{l}\text { Quadro analítico } \\
\text { Aprendizagem } \\
\text { participativa }\end{array}$ & $\begin{array}{l}\text { De Souza, H.N.a, } \\
\text { Cardoso, I.M.b, } \\
\text { de Sá Mendonça, } \\
\text { E.c, Carvalho, } \\
\text { A.F.b, de } \\
\text { Oliveira, G.B.d, } \\
\text { Gjorup, D.F.d, } \\
\text { Bonfim, V.R.d }\end{array}$ \\
\hline
\end{tabular}


Fonte: Elaborado pelos autores, 2014

\subsection{Descrição dos estudos escolhidos}

Após a análise, constatou-se a reincidência de termos associados à sustentabilidade (quadro 10): Eco, Eco-innovation, Eco-friendly, Eco-design, Ecoefficiency. Esses termos discutem de que forma estudos que envolvem a sustentabilidade na questão ambiental estão sendo tratados pela comunidade científica. Percebe-se que o prefixo Eco estabelece uma relação forte no aspecto do processo produtivo e quando associado à inovação é representado pelo termo em inglês: Eco-innovation.

O termo eco-inovação tem sido utilizado cada vez mais nas políticas de gestão ambiental das empresas e dos governos, embora em contextos e situações diversas e com variadas conotações, além disso, pode ser uma ferramenta relevante para o sucesso do sistema de inovação.

De acordo com os estudos selecionados, apesar de todos tratarem de estruturas que envolvem desenvolvimento de produtos, a eco inovação também foi apontada como relevante para soluções em produtos/serviços e infraestrutura, conforme descrito por Stoppa (2013). Buttol (2014) considera os serviços como uma forma de integração em uma rede de valor para a sustentabilidade e assume a definição de eco inovação, a partir de uma matriz de controle que se define pela sigla MEI (Medição de Eco Inovação), propondo uma abordagem incremental e comparativa baseada no ciclo de vida, o que também inclui inovações não tecnológicas. Com base nessa definição, eco-inovação está fortemente associada às formas de produção, assimilação e exploração de um produto ou serviço, levando em consideração o processo de produção ou de gestão que é adotado ou que se encontra em fase de implantação em uma organização. O uso da MEl aplica-se ao longo de seu ciclo de vida, na redução de risco ambiental, na poluição e em outros impactos negativos no que se refere à utilização de recursos (incluindo o uso de energia).

No caso em PMEs, Buttol (2014, p.1) aponta para a necessidade da ecoinovação como aperfeiçoamento contínuo e incremental de produto de modo que leva em conta todas as fases do processo (sensibilização e formação; análise do produto redesign e comunicação - e certificação). Esses processos precisam ser apoiados para superar as barreiras existentes as quais se resumem, principalmente, em falta de experiência e de recursos em PMEs.

Como formas de gestão de produtos e processos, Ferrer (2012) aponta como ferramenta de gestão ambiental a Teoria Triz, que se caracteriza como uma metodologia utilizada na engenharia de produtos e que foi desenvolvida por G. S. Altshuller durante os anos 50 (1969). Até os dias atuais, essa teoria vem sendo estudada como metodologia para soluções criativas. Seus conceitos básicos pautam-se na apresentação de solução a partir da decomposição de etapas hierárquicas, o que se dá por meio da análise da gravidade do problema, da formulação do problema e da geração de possíveis e viáveis ideias. Tais contextos podem ser comparados às metodologias de projeto de design para as quais Ferrer (2012) as propõem como uma ferramenta, a fim de aumentar a complexidade tecnológica e orientar questões ambientais de projetos de design.

Os conceitos fundamentais da TRIZ são a idealidade, a orientação à contradição e o uso de recursos existentes num sistema. Nesse sentido, o sistema pode ser entendido como a própria organização na sua relação com os seus modos de 
produção, pela forma como ocorre o processo produtivo, assim como o resultado em produtos e serviços. McAloone et al. (2010) considera, em casos bem sucedidos, como cadeia de valor e seu resultado é visto, a partir da ecoinovação. O autor apresenta uma análise descritiva das barreiras, oportunidades e mecanismos encontrados na relação entre quem produz e seus stakeholders. Com base nessas análises, a inovação em sustentabilidade é proposta na relação entre integração e identificação das questões ambientais, podendo, desse modo, ser considerada como valor na cadeia produtiva.

No processo de reconhecimento e de identificação das questões ambientais a serem admitidas como valor, é considerado segundo Reyes e Millet (2013) na organização enquanto um sistema e tratada em PMEs a partir de seu desempenho ambiental, onde o design participa, a partir da implementação de uma ferramenta de ecodesign clássico e que atua como vetor de aprendizagem dentro da empresa. Esta abordagem é chamada de "a estratégia do Cavalo de Tróia ambiental" (ETHS) e tem como estratégia subjacente o trabalho colaborativo.

Assim, no sistema, no caso de ele ser orientado para soluções em produto ou serviço, em uma abordagem como a proposta por De Souza et al (2012), leva-se em conta a aprendizagem organizacional com foco na sustentabilidade em PMEs, apresentando por meio de uma metodologia participativa, a sistematização da experiência de longo prazo de agricultores, propondo um quadro analítico que reconhece os sistemas de interações reflexivas e de aprendizagem.

Tais estudos apontam para inovações estratégicas que podem ser aplicadas em pequenas organizações, nas quais se consideram suas estruturas técnicas e seus atores sociais; bem como a forma como ocorre essa relação.

E, nesse sentido, a cadeia, conforme considerada por McAloone et al (2010) e Stoppa (2013), torna-se fundamental para a avaliação e implementação da sustentabilidade, considerando-se o impacto ambiental durante o processo produtivo, bem como a partir do comportamento de uso do produto ou serviço, assim a cadeia ou rede, passa a ser compreendida de forma ainda mais complexa considerando, quando considera-se também nessa relação os usuários como atores desse sistema.

De acordo com Stoppa (2013), a inovação por meio do design sustentável deve ser vista além do que pode ser feito e aplicado ao produto, focalizando, nessas circunstâncias, toda cadeia produtiva. Além disso, essa inovação aponta o design como uma relação virtuosa, a partir de uma visão sistêmica, considerando a necessidade de fortalecer as ferramentas conceituais e metodológicas operatórias atuais de ecodesign, de maneira a utilizar as qualidades complexas de sistemas naturais, isto é, os princípios biológicos/funções que podem ser tomados como diretrizes para aumentar a eficácia do eco-design.

Stoppa (2013) aponta para a teoria da complexidade, a teoria dos sistemas e as tecnologias emergentes como apoio em projetos sustentáveis, considerando nessas teorias, o princípio de "estoque mínimo/máximo de diversidade", pois eles destacam as qualidades complexas regidas por estratégias/funções, tais como: auto-organização, adaptabilidade, feedback, redundância, multifuncionalidade e resistência, o que, comparando aos sistemas biológicos, os permitiu sobreviver e evoluir. 
Figura 4: Diagrama: Inovação-Design-Eco-Valor

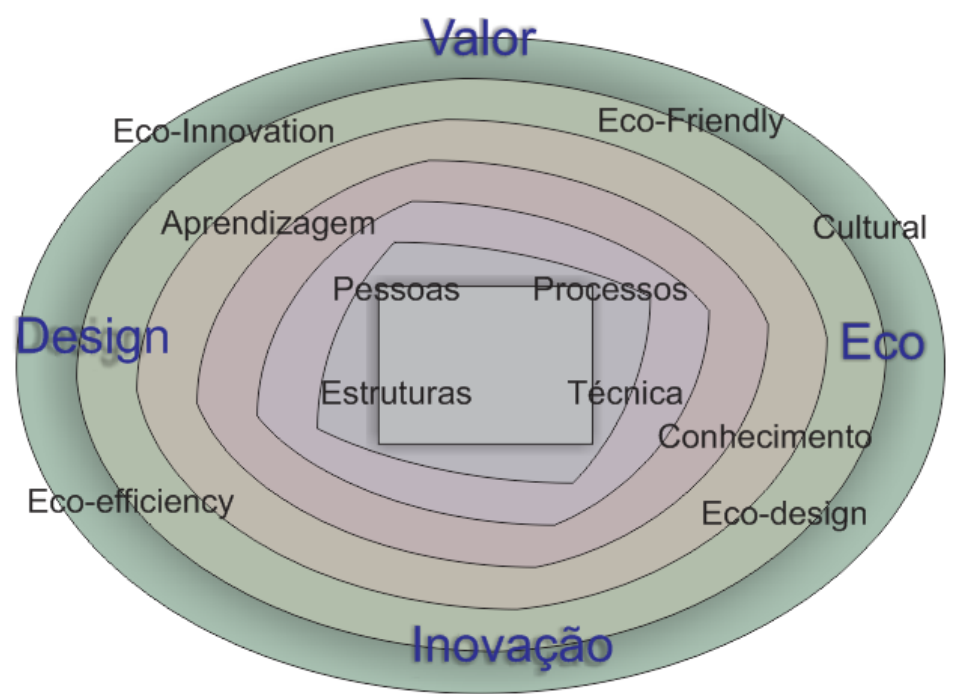

Fonte: Elaborado pelos autores, 2014

\section{RESULTADOS}

Com base nos estudos observados, verificou-se que a inovação em sustentabilidade se faz presente nas pesquisas atuais; ficou evidente também que há diferentes ferramentas estratégicas para a sua aplicação e que o design participa tanto na gestão de implementação estratégica, como em ações táticas e operacionais. Nesse sentido, em relação ao papel do design, ele é abordado como o design sendo a própria inovação, o que em todos os estudos está aliado às questões ambientais, de modo que aparecem nos trabalhos pesquisados associados ao prefixo Eco.

De acordo como os estudos aqui relacionados, a sustentabilidade ecológica apresentou-se de forma significativa, enquanto que questões sociais foram apresentadas em apenas dois dos casos, onde foram tratadas a partir da aprendizagem organizacional.

Quando o desenvolvimento sustentável é tratado de forma mais ampla, deve se levar em conta as pessoas a partir da forma como interagem com o sistema afim de promover a sua integração, para que aspectos sociais sejam fortalecidos e que possibilite a geração de valor a partir da melhoria da qualidade de vida em todos os sentidos.

Os elementos que fazem parte de uma organização, seja em caso de pequeno, médio ou grande porte, necessitam de uma abordagem sistêmica, na qual considerem se não somente as estruturas e recursos necessários para a geração de valor, mas também a cadeia, o sistema e suas relações.

De acordo com Stoppa (2013), que compara a organização a um biossistema; assim podem estar relacionados de acordo com o seu grau de complexidade para que assim possam ser definidas as suas estratégias e funções, tais como: auto-organização, adaptabilidade, feedback, redundância, multifuncionalidade e resistência.

Assim uma organização como um sistema biológico deve se considerar além de seus elementos, também suas relações e o grau como elas se estabelecem; bem como as qualidades dessas relações. 
Em uma organização vista apenas pela dimensão técnica podemos considerar seus atores a partir do resultado daquilo que produzem; porém de acordo com Vezzoli (2010) o design para sistemas deve projetar para promover a melhoria de qualidade de vida a partir de aspectos como: promover a equidade e a justiça em relação aos atores envolvidos; favorecer e integrar pessoas com necessidades especiais e marginalizadas; para que assim possibilite melhorias como a coesão e equidade social.

\section{CONSIDERAÇÕES FINAIS}

A partir desses resultados é possível estabelecer um conjunto de referências capazes de participar de matrizes estratégicas para gestão em pequenos empreendimentos, que aqui foram tratados como PMEs, bem como em outras estruturas que carecem de apoio para seu fortalecimento e para contribuir com a inserção econômica em contextos cada vez mais competitivos.

De acordo com os estudos apresentados, o design na sua relação com a sustentabilidade participa de forma mais efetiva das metodologias projetuais que envolvem o processo e o produto, porém pouco tem se falado da contribuição do design a partir de ações estratégicas, as quais envolvem aspectos sociais, sendo esse um dos eixos da sustentabilidade. Assim, aspectos como a aprendizagem podem ser eficazes na construção de valor, contribuindo com a cultura organizacional, trazendo benefícios para organização enquanto empreendimento, bem promovendo a equidade e coesão social de forma a gerar valor também para aqueles que direta ou indiretamente participam da organização. Assim o design por meio de metodologias que tenham como principio a Inovação Social, participe no processo, de forma a contribuir com o fortalecimento de uma organização, capaz de transformá-la numa cadeia de valor.

\section{REFERÊNCIAS}

BALDWIN, C. Y.; CLARK, K. B. Between "Knowledge " and " the Economy ": Notes on the Scientific Study of Designs. Scientific Studies of Designs. August. p.1-41, 2005. Disponível em: http://citeseerx.ist.psu.edu/viewdoc/download?doi=10.1.1.174.4495\&rep=rep1\&type =pdf. Acesso em 19 fev., 2014.

MOZOTA, Brigitte Borja de.( 2003). Design and competitive edge: A model for design management excellence in European SMEs. Design Management Journal:Academic Review, rev. v.2.: http:// www.dmi.org ( consultado em 10/08/2012).

MANZINI, Ezio. (2008). Design para a inovação social e sustentabilidade: Comunidades criativas, organizações colaborativas e novas redes projetuais. Epapers,. http://www.e-papers.com.br

MARTINS, R. F. F.; MERINO, E. A. D. A Gestão de Design como Estratégia Organizacional. Rosane Fonseca de Freitas, Eugenio Andrés Diáz Merino. 2. Ed. Londrina: EDUEL; Rio de Janeiro: Rio Books, 2011. 244p.

FREIRE, P. D. S.,et. al. Ferramentas de avaliação de gestão do conhecimento: um estudo bibliométrico, 16-38. International Journal of Knowledge Engineering and Management (IJKEM) ISSN 2316-6517, Florianópolis, Santa Catarina, Brasil. v. 2, n. 3, 2013. 